is Research Square

\title{
Is it Safe and Effective to Treat Complex Cervical Spondylotic Myelopathy Using Piezosurgery in Anterior Cervical Discectomy and Fusion?
}

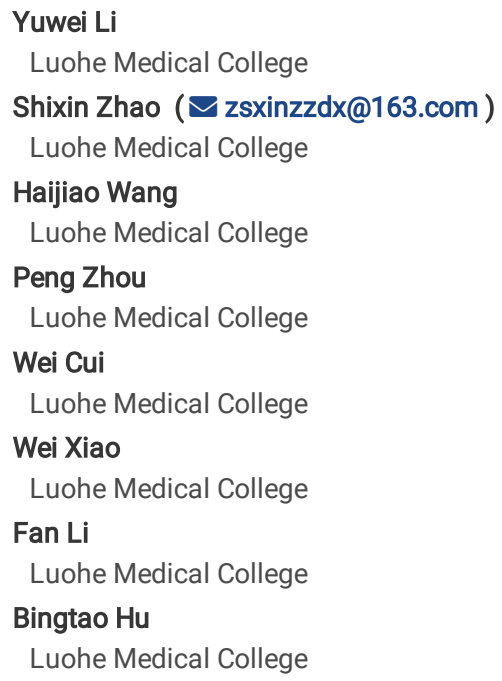




\section{Abstract}

Objective To investigate the safety and efficacy of piezosurgery in anterior cervical discectomy and fusion (ACDF) for treating patients with cervical spondylotic myelopathy (CSM) coincident retrovertebral body osteophytes adjacent to the endplate or a free nucleus pulposus migrated to the vertebral body, posteriorly; known as complex cervical spondylotic myelopathy (cCSM) here.

Methods Forty-seven patients with cCSM underwent ACDF surgery from 2014 to 2017 . Among these patients, 26 underwent ACDF using piezosurgery (group A) and 21 underwent ACDF by traditional tools such as air drill, bone curet and gun-shaped bone forceps (group B). Average operative duration, intraoperative blood loss, surgical complications, preoperative and postoperative Japanese Orthopaedic Association (JOA) scores and improvement rate were measured.

Results Average operative duration and intraoperative blood loss were significantly lower in the piezosurgery group than those in the traditional group $(P<$ 0.01). The incidences of surgical complications were $3.8 \%$ and $23.8 \%$ in the piezosurgery and traditional groups $(P<0.05)$, respectively. Whereas JOA scores and improvement rate were insignificantly different at each data collection period $(P>0.05)$; preoperative, postoperative 3 days and postoperative 1 year follow-up were included.

Conclusion For treating CCSM, both the piezosurgery and traditional tools led to significant neurological improvement. However, the piezosurgery was superior to the traditional tools in terms of operative duration, blood loss, and complication rate. Hence, the piezosurgery was a safe and effective adjunct for ACDF treating CCSM.

\section{Introduction}

Cervical spondylotic myelopathy (CSM) is characterized by progressive degeneration of the vertebral body and ligaments, resulting in spinal stenosis and subsequent compression of the spinal cord ${ }^{1,2}$. Surgery should be considered if conservative treatment fails. Anterior cervical discectomy and fusion (ACDF) has been shown to be an effective method for the treatment of $\mathrm{CSM}^{3,4}$. However, for patients with (1) large retrovertebral body osteophytes adjacent to the endplate, or (2) a free nucleus pulposus migrated to the vertebral body, posteriorly; known as complex cervical spondylotic myelopathy (cCSM) here; a surgeon may consider anterior cervical corpectomy and fusion (ACCF) ${ }^{5,6}$. In this case, ACDF may be suboptimal due to the higher chance of incomplete decompression posterior to the mid-vertebral body and limited visual access during the surgery ${ }^{5,7}$.

A majority of studies have endorsed the clinical outcomes of ACCF treatment for CCSM. Some studies have indicated, however, that ACCF is associated with a higher incidence of complications including a wider range of operations, and an increased risk of prosthesis extrusion due to the fewer possible points for ventral plate screw fixation ${ }^{8,9}$. With the application of piezosurgery in cervical surgery, the author found that for cCSM with the soft free nucleus pulposus no more than $1 / 2$ and osteophytes no more than $1 / 3$ of the vertebral body posteriorly, can ACDF completely decompress cCSM through a narrow intervertebral space? If that hypothesis is confirmed, the application of ACCF is reduced to minimize the risk of prosthesis extrusion, shorten the fusion distance, and decrease the risk of nerve damage.

To date, it is unknown as to the safety and efficacy of ACDF in the treatment of cCSM with piezosurgery. Moreover, few studies have compared clinical outcomes between piezosurgery and traditional tools in ACDF for CCSM. The purpose of this study was to assess the differences in peri- and 1-year postoperative outcomes associated with piezosurgery versus traditional tools for the treatment of cCSM.

\section{Methods}

\section{Patients Population}

Forty-seven consecutive patients treated with ACDF for single segment CCSM in our institution from 2014 to 2017 . Among these patients, 26 were treated using the piezosurgery for decompression (group A), whereas 21 were subjected to traditional tools such as air drill, bone curet and gun-shaped bone forceps (group B). The inclusion criteria for this study were as follows: (1) diagnosed as CSM, including medical history, physical examination, and imaging examinations. (2) Free nucleus pulposus and/or posterior osteophytes of the vertebral body are limited to one segment, according to the aims of this study, patients with 2 or multi-level cCSM ( $\geq 2$ levels) who fail to meet the inclusion criteria. (3) Those who had a previous history of cervical surgery were excluded. (4) The lesions were between C3/4 and C6/7. (5) The free nucleus pulposus does not exceed 1/2 of the vertebral body, and osteophytes do not exceed 1/3 of the vertebral body, posteriorly (Fig.1). Patients were divided into two groups according to intraoperative decompression tools: group A (26 cases) using piezosurgery; group B (21 cases) using traditional tools. There were no significant differences in sex, age, BMI, involved lesions and Japanese Orthopaedic Association score (JOA score) between the two groups $(P>0.05$; Table 1$)$.

The study protocol was approved by the ethics board of Luohe Central Hospital. Surgical procedures and perioperative management of all patients strictly followed the relevant guidelines and regulations. We stated that informed consent was obtained from all participants and/or their legal guardians. Patients outcomes were initially collected independently from patients with informed consent and then analyzed blindly to avoid influencing the outcome scores.

\section{Surgical Technique}

All patients were taken supine position under general anesthesia. The right transverse incision was taken and separated until the vertebral body was exposed. After removing the intervertebral disc, properly open the intervertebral space.

Group A: All patients were treated with piezosurgery for decompression. First, the cortical bone and/or osteophytes at the posterior edge of the vertebral body were scraped with the long-handled direct curet, and then the curved curet was attached to the posterior longitudinal ligament (PLL) and placed in the posterior 
edge of the vertebral body, which was further scraped until the posterior margin of the intervertebral space forms a trapezoid narrow in front and wide in back. The PLL attachment on vertebral body was disconnected in this enlarged space, this is followed by the detection and removal of the compressors in front of the spinal dural. The schematic of ACDF using piezosurgery is shown in figure 2.

Group B: All patients had the posterior edge of the vertebral body thinned with the air drill, the bone cortex and/or osteophytes at the posterior margin of the vertebral body were then removed using a conventional curet or gun-shaped bone forceps, the posterior trapezoid of the intervertebral space is considered as complete decompression. The PLL was removed, and the compressors were explored and removed under direct vision.

In the 2 groups, zero-P was implanted in the intervertebral space after complete decompression, and the incision was closed after intraoperative anteroposterior and lateral X-ray confirmed that the prosthesis is in good position. All the patients were allowed to get out of bed the next day after the operation and perform limb rehabilitation exercise.

\section{Outcome measures}

Observation indicators included general data, neurological function indicators, and radiological data.

The general indicators consist of operation time, intraoperative blood loss, and surgical complications including spinal cord or nerve root injury, cerebrospinal fluid leakage, injury of superior laryngeal nerve or recurrent laryngeal nerve, dyspnea, dysphagia, hematoma, C5 nerve root paralysis, infection of surgical incision, pulmonary and urinary tract infection, etc.

The nerve functions were evaluated by the JOA scores and the neurological function improvement rate was calculated.

The improvement rate $=\frac{\text { follow up score-preoperative score }}{17 \text {-preoperative score }} \times 100 \%$

The radiological indicators including X-ray, computed tomography (CT), and magnetic resonance imaging (MRI) were reviewed assessing the preoperative, postoperative and final follow-up status, evaluating the degree of preoperative compression and the effect of postoperative decompression as well as prosthesis position.

\section{Statistical analysis}

SPSS software (version 22) was performed for statistical analysis. The results were presented as average \pm SD. The age, BMI, duration of surgery, intraoperative blood loss and JOA score were analyzed using independent sample t test, whereas gender, surgical segment, postoperative complications and JOA improvement rate were assessed by $x 2$ test. $P<0.05$ was considered statistically significant.

\section{Results}

The surgeries of all patients in the 2 groups completed successfully. The last follow-up was set at 1 year post-operatively. In terms of the demographic data of the two groups, no significant statistical difference was found (Table 1). Typical cases data were shown in Figure 3.

\section{Table 1 Comparison of general data between the two groups}

\begin{tabular}{|c|c|c|c|c|c|c|c|c|c|}
\hline \multirow[t]{2}{*}{ Group } & \multirow[t]{2}{*}{ Number } & \multicolumn{2}{|c|}{ Gender } & \multirow[t]{2}{*}{ Age, mean(years) } & \multirow[t]{2}{*}{ BMI } & \multicolumn{4}{|c|}{ Operative level } \\
\hline & & $M$ & W & & & $\mathrm{C}_{3 / 4}$ & $\mathrm{C}_{4 / 5}$ & $\mathrm{C}_{5 / 6}$ & $\mathrm{C}_{6 / 7}$ \\
\hline Group A & 26 & 15 & 11 & $54.00 \pm 9.75$ & $24.12 \pm 5.06$ & 2 & 7 & 11 & 6 \\
\hline Group B & 21 & 11 & 10 & $49.33 \pm 8.05$ & $24.26 \pm 5.45$ & 2 & 6 & 8 & 5 \\
\hline Statistics & & \multicolumn{2}{|c|}{$\chi^{2}=0.133$} & $t=1.761$ & $t=0.091$ & \multicolumn{4}{|c|}{$\chi 2=0.111$} \\
\hline$P$ & & \multicolumn{2}{|c|}{0.716} & 0.085 & 0.928 & \multicolumn{4}{|c|}{0.991} \\
\hline
\end{tabular}

\section{General indicators}

The operation time was $45.7 \pm 3.9 \mathrm{~min}$ and $52.7 \pm 6.7 \mathrm{~min}$ in the group A and group B, respectively. In group A, the intraoperative blood loss was $48.9 \pm 4.4 \mathrm{ml}$, compared with the intraoperative blood loss of $117.9 \pm 16.3 \mathrm{ml}$ in group B. There were significant differences between the two groups in the operation time and intraoperative blood loss $(P<0.05)$. In terms of complications, only 1 patient in group A had urinary tract infection, no cerebrospinal fluid leakage or spinal cord injury occurred, while 2 patients in group B had cerebrospinal fluid leakage, 1 had hoarseness, 2 had swallowing discomfort and no spinal cord injury occurred. A statistically significant difference was found between the two groups $(P<0.05$; Table 2$)$.

\section{Neurological function indicators}

The preoperative JOA scores were $7.2 \pm 0.9$ with postoperatively to $11.3 \pm 1.2$ (JOA improvement rate was $39.0 \%$ ), improved to $14.6 \pm 1.8$ at final follow-up (JOA improvement rate was $71.6 \%$ ) in group A. The preoperative JOA scores were $7.4 \pm 1.0$ with postoperatively to $11.6 \pm 1.1$ (JOA improvement rate was $38.7 \%$ ), improved to $14.6 \pm 1.9$ at final follow-up (JOA improvement rate was $69.4 \%$ ) in group B. There was no significant difference between two groups in the JOA 
scores and JOA improvement rate for neurofunctional improvement at all time points $(P>0.05)$. However, the postoperative and the last follow-up were significantly improved in both groups compared with that pre-operation $(P<0.001$; Table 2$)$.

Table 2 Comparison of two groups of operation time, intraoperative blood loss, neurological function and complications

\begin{tabular}{|c|c|c|c|c|c|c|c|c|c|c|}
\hline \multirow[t]{2}{*}{ Group } & \multirow[t]{2}{*}{ Number } & \multirow{2}{*}{$\begin{array}{l}\text { Operation } \\
\text { time (min) }\end{array}$} & \multirow{2}{*}{$\begin{array}{l}\text { Intraoperative } \\
\text { blood loss } \\
(\mathrm{ml})\end{array}$} & \multirow[t]{2}{*}{ Complications } & \multicolumn{4}{|l|}{ JOA scores } & \multicolumn{2}{|c|}{ JOA improvement ré } \\
\hline & & & & & Preoperative & Postoperative & $\begin{array}{l}\text { Last } \\
\text { follow-up }\end{array}$ & $\begin{array}{l}\text { F and } P \\
\text { value }\end{array}$ & Postoperative & $\begin{array}{l}\text { La: } \\
\text { fol } \\
\text { up }\end{array}$ \\
\hline \multirow[t]{2}{*}{ Group A } & 26 & $45.70 \pm 3.93$ & $48.85 \pm 4.38$ & 1 & $7.20 \pm 0.91$ & $11.31 \pm 1.20$ & $14.61 \pm 1.80$ & $F=195.549$ & $39.0 \%$ & 71. \\
\hline & & & & & & & & $P<0.001$ & & \\
\hline \multirow[t]{2}{*}{ Group B } & 21 & $52.74 \pm 6.71$ & $117.86 \pm 16.32$ & 5 & $7.38 \pm 1.00$ & $11.55 \pm 1.08$ & $14.57 \pm 1.89$ & $F=143.315$ & $38.7 \%$ & 69. \\
\hline & & & & & & & & $P<0.001$ & & \\
\hline Statistics & & $t=4.254$ & $t=18.835$ & $\chi^{2}=4.157$ & $t=0.611$ & $t=0.703$ & $t=0.061$ & & $x^{2}=0.686$ & $x^{2}=$ \\
\hline$P$ & & 0.000 & 0.000 & 0.041 & 0.544 & 0.485 & 0.951 & & 0.496 & 0.2 \\
\hline
\end{tabular}

\section{Discussion}

One of the most serious complications of anterior cervical surgery is spinal cord injury. Traditional decompression tools including conventional curet and gunshaped bone forceps have the potential risk of squeezing the dura, while air drill have the risk of scraping the surrounding soft tissue and damaging the spinal dural $^{10-13}$. One main advantage of the piezosurgery over the traditional tools is that the former produces mechanical vibration (amplitude $0.05 \sim 0.36 \mathrm{~mm}$ ) on bone with vibration frequency of $22.5 \mathrm{khz} \sim 40 \mathrm{kHz}$, so as to achieve easy cutting effect. The amplitude, meanwhile, is obviously lower than the elastic limit of the spinal dural, which can absorb the vibration energy through elastic vibration, so as to avoid spinal cord injury $6,14,15$. Some studies also supported the above views in which Nakagawa showed that the use of piezosurgery could reduce the occurrence of mechanical injuries of spinal cord ${ }^{16}$, while Nakase confirmed that the ultrasonic dynamic system was more suitable for the operation of delicate structures ${ }^{17}$. In this study, osteophytes at the posterior edge of the vertebral body were removed by using an piezosurgery to make the intervertebral space into a trapezoidal shape, fully relieving the spinal cord compression and ensuring safety at the same time. No device-related dural tear or spinal cord injury was found in patients in group A; cerebrospinal fluid leakage, however, occurred in 2 patients in group B.

In addition to the neuroprotective advantages, the reduction of intraoperative bleeding is another advantage of piezosurgery. Sanbom ${ }^{18}$ found that the ultrasonic dynamic system significantly reduced the amount of blood loss in the process of bone cutting compared with traditional tools, based on its ultrasonic cavitation effect ${ }^{19,20}$. The results of this study showed that the operative time and intraoperative blood loss in group A were significantly less than those in group $B$.

Anterior cervical surgery, including ACDF and ACCF, is the mainstream surgical method for the treatment of CSM with localized compression lesions ${ }^{21-23}$. Both of them can effectively remove intervertebral discs and retrovertebral body osteophytes ${ }^{24,25}$. Although the former has less damage to the normal structure of cervical vertebra and less intraoperative blood loss than the latter while fully decompressing. Nevertheless, due to the limitation of ACDF in the narrow operating space, confined surgical field and difficulty in hemostasis, the risk of spinal cord injury is higher than that of ACCF, especially in the patients with CSM coincident large posterior osteophytes adjacent to the endplate or a free nucleus pulposus migrated to the rear of the vertebral body ${ }^{26}$. Therefore, in consideration of safety and decompression, most researchers adopted ACCF for patients with large osteophytes or free nucleus pulposus in the posterior vertebral body. One main advantage of piezosurgery is that ACCF is not required for these patients as piezosurgery can easily remove the bone of the vertebral body until the intervertebral space presents a trapezoidal shape with narrow front and wide back, which expands the surgical field and operating space, then can safely and effectively dislodge osteophytes and free nucleus to relieve spinal cord compression ${ }^{27}$. As a revolutionary surgical tool in the field of spinal surgery, some clinical studies have reported that ultrasonic osteodynamic system can improve surgical efficiency, shorten surgical time, reduce bleeding and ensure neurosafety ${ }^{18-20}$. Even when large osteophytes and free nucleus pulposus are encountered, CCSM could still be treated with ACDF instead of ACCF with the aid of piezosurgery.

Several limitations of this retrospective study should be noted. The small sample of patients in our study, being monocentric, may have affected the results of this study. Moreover, the last follow-up time of all patients was relatively short. Polycentric data with larger sample of patients are urged to further clarify the safety and efficiency of piezosurgery.

\section{Conclusion}

This study confirmed that for patients with cCSM combined with large osteophytes or free nucleus pulposus, the use of piezosurgery can enlarge the surgical indications of ACDF and avoid ACCF to reduce the incidence of prosthesis related complications. Although either the piezosurgery or traditional tools resulted in significant neurological improvement, nevertheless the former was superior to the latter in terms of operative time, intraoperative blood loss, and surgical complications. The piezosurgery may be a better candidate for treating CCSM combined with large osteophytes or free nucleus pulposus compared with the traditional tools. 


\section{Declarations}

\section{Acknowledgements}

This work was financed by grants from Henan Provincial Health and Family Planning Commission (LHGJ20191424).

\section{Author Contributions}

Yuwei Li and Haijiao Wang conceived and designed the study. Peng Zhou, Wei Cui and Wei Xiao collected the data. Fan Li analyzed the data. Bingtao Hu performed the statistical analyses. Shixin Zhao wrote the manuscript. All authors approved the current manuscript to be published, attested that they contributed substantially to the current work, and disclosed that there was no writing assistance.

\section{Statement on research methods}

The study protocol was approved by the ethics board of Luohe Central Hospital. Surgical procedures and perioperative management of all patients strictly followed the relevant guidelines and regulations. We stated that informed consent was obtained from all participants and/or their legal guardians. Patients outcomes were initially collected independently from patients with informed consent and then analyzed blindly to avoid influencing the outcome scores.

\section{Additional Information}

Competing financial interests: The authors declare no competing financial interests.

\section{References}

1. Nouri, A., Tetreault, L., Singh, A., Karadimas, S. K., \& Fehlings, M. G. Degenerative Cervical Myelopathy: Epidemiology, Genetics, and Pathogenesis. Spine, 40(12), E675-E693 (2015)

2. Tetreault, L., Goldstein, C. L., Arnold, P., Harrop, J., Hilibrand, A., Nouri, A., \& Fehlings, M. G. Degenerative Cervical Myelopathy: A Spectrum of Related Disorders Affecting the Aging Spine. Neurosurgery, 77 Suppl 4, S51-S67 (2015).

3. Qizhi, S., Peijia, L., Lei, S., Junsheng, C., \& Jianmin, L. Anterior cervical discectomy and fusion for noncontiguous cervical spondylotic myelopathy. Indian journal of orthopaedics, 50(4), 390-396 (2016).

4. Liow, M., Lee, M., Goh, G. S., Chen, L., Yue, W. M., Guo, C. M., \& Tan, S. B. Poorer Fusion Outcomes in Diabetic Cervical Spondylotic Myelopathy Patients Undergoing Single-level Anterior Cervical Discectomy and Fusion Does Not Compromise Functional Outcomes and Quality of Life. Spine, 43(7), 477-483 (2018).

5. Niedzielak, T. R., Palmer, J., \& Malloy, J. P., 4th. Clinical Comparison of Surgical Constructs for Anterior Cervical Corpectomy and Fusion in Patients With Cervical Spondylotic Myelopathy or Ossified Posterior Longitudinal Ligament: A Systematic Review and Meta-Analysis. Clinical spine surgery, 31(6), 247260 (2018).

6. Okada, K., Shirasaki, N., Hayashi, H., Oka, S., \& Hosoya, T. Treatment of cervical spondylotic myelopathy by enlargement of the spinal canal anteriorly, followed by arthrodesis. The Journal of bone and joint surgery. American volume, 73(3), 352-364 (1991).

7. Deora, H., Kim, S. H., Behari, S., Rudrappa, S., Rajshekhar, V., Zileli, M., Parthiban, J., \& World Federation of Neurosurgical Societies (WFNS) Spine Committee. Anterior Surgical Techniques for Cervical Spondylotic Myelopathy: WFNS Spine Committee Recommendations. Neurospine, 16(3), 408-420 (2019).

8. Wang, T., Wang, H., Liu, S., An, H. D., Liu, H., \& Ding, W. Y. Anterior cervical discectomy and fusion versus anterior cervical corpectomy and fusion in multilevel cervical spondylotic myelopathy: A meta-analysis. Medicine, 95(49), e5437 (2016).

9. Banno, F., Zreik, J., Alvi, M. A., Goyal, A., Freedman, B. A., \& Bydon, M. Anterior Cervical Corpectomy and Fusion Versus Anterior Cervical Discectomy and Fusion for Treatment of Multilevel Cervical Spondylotic Myelopathy: Insights from a National Registry. World neurosurgery, 132, e852-e861 (2019).

10. Grauvogel, J., Scheiwe, C., \& Kaminsky, J. Use of Piezosurgery for removal of retrovertebral body osteophytes in anterior cervical discectomy. The spine journal : official journal of the North American Spine Society, 14(4), 628-636 (2014).

11. Rhee, J. M., \& Ju, K. L. Anterior Cervical Discectomy and Fusion. JBJS essential surgical techniques, 6(4), e37 (2016).

12. Tasiou, A., Giannis, T., Brotis, A. G., Siasios, I., Georgiadis, I., Gatos, H., Tsianaka, E., Vagkopoulos, K., Paterakis, K., \& Fountas, K. N. Anterior cervical spine surgery-associated complications in a retrospective case-control study. Journal of spine surgery (Hong Kong), 3(3), 444-459 (2017).

13. Park, M. S., Moon, S. H., Lee, H. M., Kim, S. W., Kim, T. H., Min, S. K., Suh, B. K., \& Riew, K. D. Do Anterior Cervical Osteophytes Prevent Dynamization of Cervical Dynamic Plates?. Clinical spine surgery, 29(6), E314-E318 (2016).

14. Pan, S. F., \& Sun, Y. Application of Piezosurgery in Anterior Cervical Corpectomy and Fusion. Orthopaedic surgery, 8(2), 257-259 (2016).

15. Li, K., Zhang, W., Li, B., Xu, H., Li, Z., Luo, D., Zhang, J., \& Ma, J. Safety and efficacy of cervical laminoplasty using a piezosurgery device compared with a high-speed drill. Medicine, 95(37), e4913 (2016).

16. Nakagawa, H., Kim, S. D., Mizuno, J., Ohara, Y., \& Ito, K. Technical advantages of an ultrasonic bone curette in spinal surgery. Journal of neurosurgery. Spine, 2(4), 431-435 (2005).

17. Nakase, H., Matsuda, R., Shin, Y., Park, Y. S., \& Sakaki, T. The use of ultrasonic bone curettes in spinal surgery. Acta neurochirurgica, 148(2), 207-213 (2006). 
18. Sanborn, M. R., Balzer, J., Gerszten, P. C., Karausky, P., Cheng, B. C., \& Welch, W. C. Safety and efficacy of a novel ultrasonic osteotome device in an ovine model. Journal of clinical neuroscience : official journal of the Neurosurgical Society of Australasia, 18(11), 1528-1533 (2011).

19. Matsumoto, S., Kawabe, N., Mizuno, Y., Shirasugi, N., Suzuki, H., \& Umemoto, S. The ultrasonic trocar provides an easy, sharp, bloodless, and repeatable approach to the abdominal cavity. JSLS : Journal of the Society of Laparoendoscopic Surgeons, 6(4), 401-405 (2002).

20. Timothy, J., Petralia, V., \& Wilson, J. R. Use of an Ultrasonic Bone Curet for the Extraction of a Cervical Artificial Disc: A Novel Application: A Case Report. JBJS case connector, 8(1), e5 (2018).

21. Puvanesarajah, V., Jain, A., Cancienne, J. M., Shimer, A. L., Singla, A., Shen, F., \& Hassanzadeh, H. Complication and Reoperation Rates Following Surgical Management of Cervical Spondylotic Myelopathy in Medicare Beneficiaries. Spine, 42(1), 1-7 (2017).

22. Salem, H. M., Salem, K. M., Burget, F., Bommireddy, R., \& Klezl, Z. Cervical spondylotic myelopathy: the prediction of outcome following surgical intervention in 93 patients using T1- and T2-weighted MRI scans. European spine J, 24(12), 2930-2935 (2015).

23. Park, M. S., Ju, Y. S., Moon, S. H., Kim, T. H., Oh, J. K., Makhni, M. C., \& Riew, K. D. Reoperation Rates After Anterior Cervical Discectomy and Fusion for Cervical Spondylotic Radiculopathy and Myelopathy: A National Population-based Study. Spine, 41(20), 1593-1599 (2016).

24. Witiw, C. D., Tetreault, L. A., Smieliauskas, F., Kopjar, B., Massicotte, E. M., \& Fehlings, M. G. Surgery for degenerative cervical myelopathy: a patientcentered quality of life and health economic evaluation. The spine journal : official journal of the North American Spine Society, 17(1), 15-25 (2017).

25. Banno, F., Zreik, J., Alvi, M. A., Goyal, A., Freedman, B. A., \& Bydon, M. Anterior Cervical Corpectomy and Fusion Versus Anterior Cervical Discectomy and Fusion for Treatment of Multilevel Cervical Spondylotic Myelopathy: Insights from a National Registry. World neurosurgery, 132, e852-e861 (2019).

26. Mao, N., Wu, J., Zhang, Y., Gu, X., Wu, Y., Lu, C., Ding, M., Lv, R., Li, M., \& Shi, Z. A Comparison of Anterior Cervical Corpectomy and Fusion Combined With Artificial Disc Replacement and Cage Fusion in Patients With Multilevel Cervical Spondylotic Myelopathy. Spine, 40(16), 1277-1283 (2015).

27. Oh, M. C., Zhang, H. Y., Park, J. Y., \& Kim, K. S. Two-level anterior cervical discectomy versus one-level corpectomy in cervical spondylotic myelopathy. Spine, 34(7), 692-696 (2009).

\section{Figures}
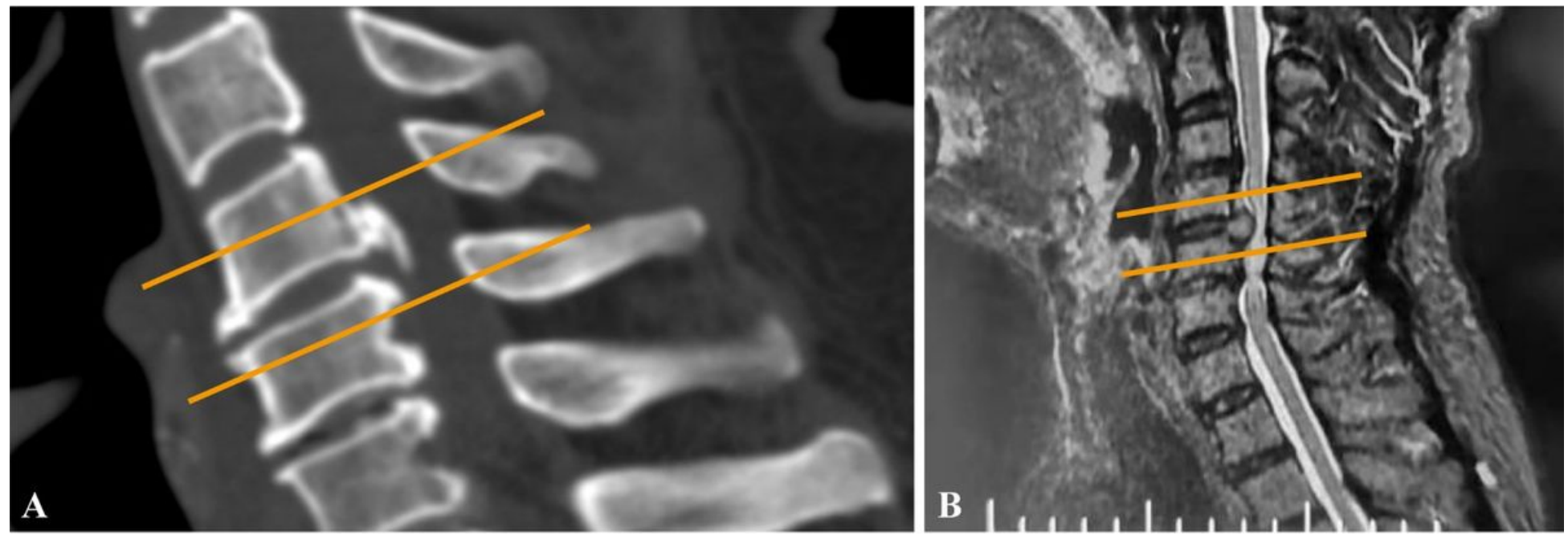

Figure 1

Case inclusion criteria: A. Osteophytes no more than one-third of vertebral body height or B. Nucleus pulposus should not exceed half of vertebral body height

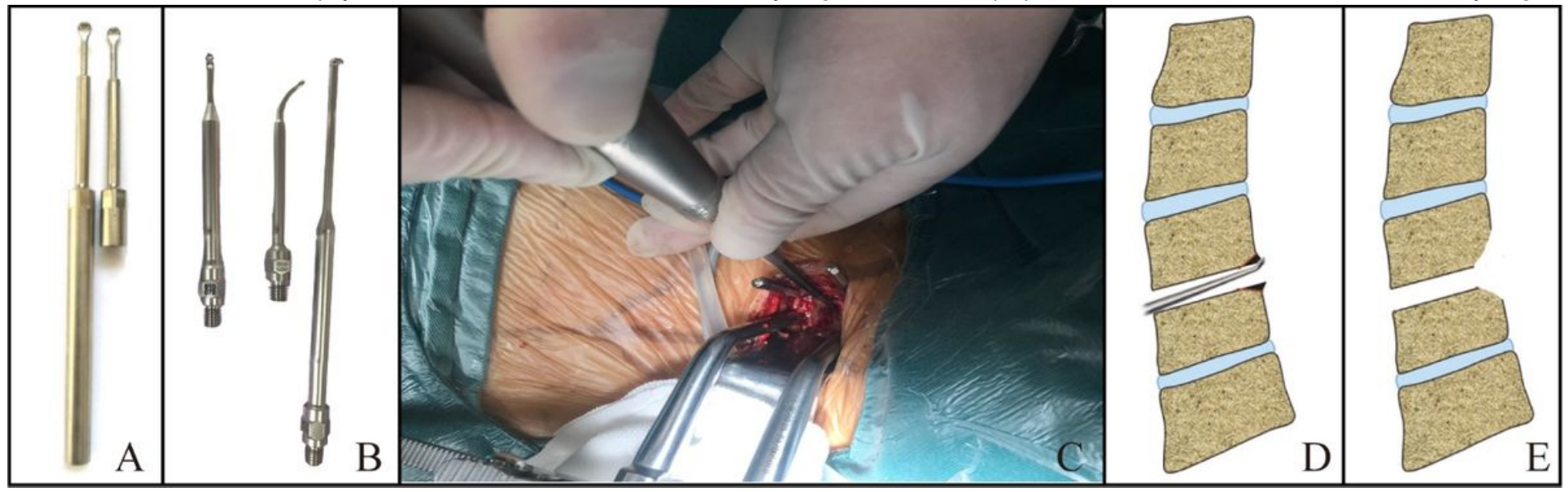

Figure 2 
Diagram of piezosurgery operation: A. straight piezosurgery; B. piezosurgery with different angles and shapes; C. piezosurgery was used to remove osteophytes from the posterior margin of vertebral body; D and E. piezosurgery was used to remove osteophytes behind the vertebral body until the posterior intervertebral space became a trapezoid with narrow front and wide back.

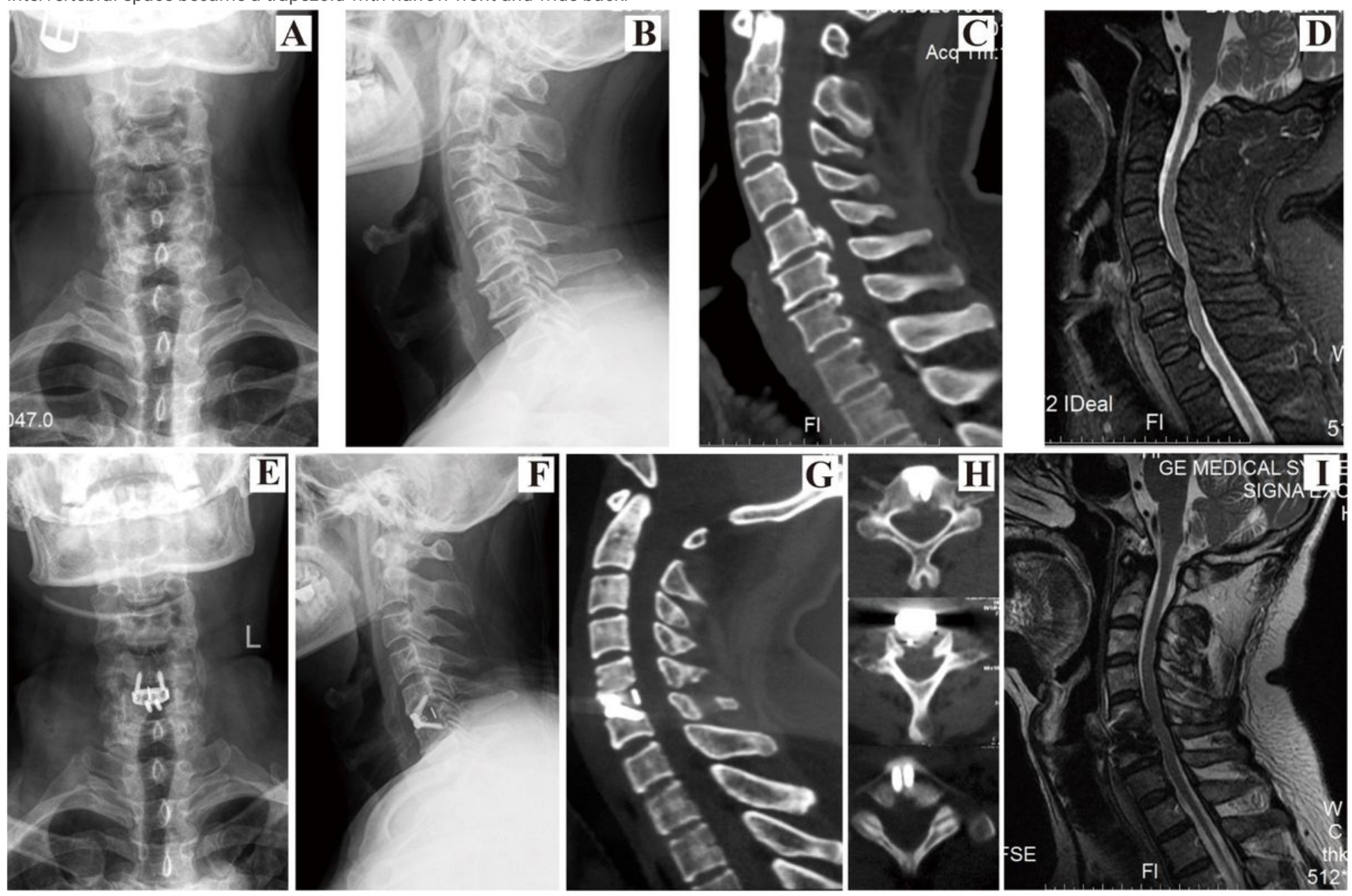

Figure 3

56 year-old man with CSM treated with ACDF using piezosurgery. A and B. Preoperative anteroposterior and lateral X-ray images showed the presence of large osteophytes in the c5/6 vertebral bodies; C. Preoperative sagittal CT examination showed c5/6 intervertebral disc herniation with calcification; D. Preoperative sagittal MRI revealed the $\mathrm{c} 5 / 6$ disc herniation that compressed the spinal cord; $\mathrm{E}$ and F. Postoperative anteroposterior and lateral X-ray examination showed that the prosthesis was in good position and the posterior vertebral osteophytes had disappeared; $\mathrm{G}$ and H. Postoperative axial sagittal CT showed c5/6 posterior osteophytes were removed completely and cervical canal area was significantly increased; I. Postoperative sagittal T2-weighted MRI showed relief of c5/6 spinal cord compression. 